\title{
Fasol, Laura: Stadtgestalt und Stadtgesellschaft. Identitätskonstruktionen in Winterthur, Luzern und Bern um 1900, 288 S., Chronos Verlag, Zürich 2020.
}

\section{Dieter Schott}

Angenommen: 13. April 2021 / Online publiziert: 28. April 2021

(C) Der/die Autor(en) 2021

Die Druckfassung der 2017 bei Jon Mathieu an der Universität Luzern angenommenen Dissertation von Laura Fasol will die Konstruktion von Stadtidentitäten untersuchen, die sich in Reaktion auf Prozesse der Modernisierung und typologischen Diversifizierung seit der zweiten Hälfte des 19. Jahrhunderts in Schweizer Städten vermehrt veränderten. Zugleich eröffnet sie ihre Einleitung mit einem Verweis auf „Paris als Hauptstadt des 19. Jahrhunderts“ im Hinblick auf den bahnbrechenden Stadtumbau durch den Präfekten Georges-Eugene Haussmann in den 1850er und 1860er Jahren und dessen Strahlkraft auch auf andere europäische Städte. Von der Feststellung ausgehend, dass die städtebaulichen Veränderungen des 19. Jahrhunderts, etwa die Anlage von Bahnhöfen, die Niederlegung der Befestigungen, Auswirkungen auf die mentalen Bilder von Stadt hatten, fragt Fasol danach, wie diese Veränderungen die Wahrnehmung von Städten, die Darstellung von Städten und die Konstruktion von Stadtidentitäten beeinflussten. Zugleich stellt sie auch die Umkehrfrage, wie Stadtwahrnehmungen, Stadtdarstellungen und Stadtidentitäten Stadtgestalt und Stadtgesellschaft beeinflussten. Fasol geht von einem im Vergleich zu den europäischen Metropolen verzögert eintretenden und weniger radikalen Stadtumbau Schweizer Städte aus, stellt allerdings fest, dass die relative Ähnlichkeit Schweizer Städte, die man noch um die Mitte des 19. Jahrhunderts beobachten konnte, in der zweiten Jahrhunderthälfte verloren ging und sich klar unterscheidbare Stadttypen herausbildeten. Als Repräsentanten solcher Typen hat Fasol Winterthur für den Typus der Industriestadt, Luzern für eine Touristenstadt und Bern als Bundesstadt/ Hauptstadt ausgewählt, eine Wahl, die insgesamt überzeugen kann. Die Schweizer Urbanisierung sieht Fasol als im europäischen Vergleich etwas verzögert, wobei gegen Ende des 19. Jahrhunderts eine deutliche Beschleunigung einsetzte; während die Gesamtbevölkerung der Schweiz zwischen 1888 und 1910 um ein Drittel wuchs, stieg die Stadtbevölkerung um 73,5 Prozent. Gleichwohl war die Stadtlandschaft der Schweiz im europäischen Vergleich von relativ vielen, aber eher kleinen Städten gekennzeichnet, Metropolen wie Paris, London oder Berlin gab es keine. So hatte

D. Schott $(\triangle)$

TU Darmstadt, Darmstadt, Deutschland

E-Mail: schott@pg.tu-darmstadt.de 
Winterthur mitsamt Vororten 191046.000 Einwohner, Luzern zählte knapp 40.000, Bern gut 90.000 Einwohner.

Konzeptionell beansprucht Fasol, in Anlehnung an Bruno Fritzsche und François Walter ,eine Schweizer Stadtgeschichte zu schreiben, die mehr ist als nur eine ,urban biography“" (S. 19f.), verfolgt allerdings im Unterschied zu Fritzsche und Walter einen kulturhistorischen Ansatz anhand von drei Fallbeispielen. Sie schließt an Forschungsstränge vor allem auch in der deutschen Stadtgeschichte an, die sich mit ,Repräsentationen von Städten“ auseinandergesetzt haben, explizit werden die Arbeiten von Jochen Guckes und Sandra Schürmann genannt. Es geht also um ,Images $^{6}$, um die Konstruktion von Fremd- und Selbstbildern und deren Wandel. Fasol fragt nach der ,offiziellen Imagepolitik‘ der Städte, zugleich aber auch nach Gegenbildern, nach Kritik daran. Die Quellen, die Fasol für alle drei Städte auswertet, sind einmal Stadtführer und Reiseführer, sowie von offizieller Seite geförderte Darstellungsschriften. Zweitens setzt sie sich ausführlich mit der ikonografischen Tradition der Städte, zurückgehend meist bis ins 16. Jahrhundert, auseinander. Sie zeigt an klassischen Stadtveduten, etwa von Merian, dass der Blick auf die Beispielstädte sich meist in der zweiten Hälfte des 19. Jahrhunderts signifikant veränderte. In dem Maße, wie sich der zunächst vornehmlich von Engländern praktizierten Alpentourismus verstärkte, wurde es immer wichtiger, den Bezug zur Landschaft, konkret die Alpenkulisse, mit ins (Stadt-)Bild aufzunehmen. Dies bedeutete jedoch meist, dass die klassische Perspektive, die bemüht war, die Stadt von der "Schokoladenseite", mit den eindrucksvollsten Gebäuden und Mauerteilen im Vordergrund zu präsentieren, aufgegeben werden musste, und der Blick auf die Stadt so gewählt wurde, um den Landschaftsbezug zu unterstreichen.

Innovativ ist das Beiziehen einer größeren Zahl von Postkarten in Ergänzung zu den offiziellen und aufwändigen Stadtbildern, wobei vor allem im Kapitel zu Winterthur Fasol auch interessante kommunikationsgeschichtliche Beobachtungen zur Funktion der Verwendung dieser Postkarten macht, die etwa der heutigen Nutzung von Textnachrichten oder Telefon nahekam.

Fasol stellt jeweils ihre Beispielstadt in einem ,Stadtporträt' kurz mit den wesentlichen Charakteristika, auch illustriert mit Stadtplänen, vor, analysiert dann die Stadtrepräsentationen anhand offizieller Stadtführer und Reiseführer, geht auf Kritik an den offiziellen Images ein, etwa die Perspektive von städtischen Kräften, die - in Winterthur - der Industrie kritisch gegenüberstanden oder - in Luzern - den starken Fokus auf Tourismus kritisierten. Die Gliederungsstruktur wird je nach Quellenlage angepasst; so umfasst Kapitel II zu Winterthur eine ausführliche Auseinandersetzung mit Postkarten, während im Luzerner Kapitel ein Konflikt um den möglichen Abbruch der Kapellbrücke als mikrohistorische Vertiefung näher durchleuchtet wird. Die vergleichende Perspektive wird dann einerseits im Kapitel zu Winterthur durch den Vergleich mit der Imagepolitik deutscher Städte wie Dresden oder Dortmund hergestellt, andererseits innerhalb ihres Samples durch den Vergleich der Naturdarstellung in der Stadtidentität von Winterthur und Luzern. Eine Fallstudie unternimmt Fasol auch zum Projekt der Kirchenfeldbrücke in Bern, wo sie exemplarisch Konflikte zwischen der Burgergemeinde, Besitzerin des durch die Brücke erschlossenen Areals jenseits der Altstadt, und den, die städtische Modernisierung vorantreibenden, Liberalen untersucht. 
In ihrem Fazit unterstreicht Fasol nochmals den typologischen Wandel ihrer Beispielstädte und den damit verbundenen raumstrukturellen Wandel: Der Schwerpunkt der Stadt verschob sich meist weg von der Altstadt hin zum Bahnhof, beziehungsweise in Luzern zum Touristenquartier am See. In Bern blieb zwar die Altstadt insgesamt weiter das Identitätszentrum, es fand jedoch eine Verlagerung weg von der kleingewerblichen unteren Stadt, einst das Zentrum, hin zum Areal in der Nähe von Bahnhof und Bundeshaus statt. Die Konstruktion von Stadtidentitäten hat drei gemeinsame Themen, ,der Bezug auf die Geschichte, den Umgang mit Modernisierung und die Hervorhebung der Natur" (S. 263). Für Winterthur und Luzern stellt Fasol eine relativ klare und auch wenig kontroverse Stadtidentität fest. Winterthur präsentiert sich als moderne Industriestadt, die gleichwohl in Natur eingebettet sei und die Übel moderner Industrie vermeiden beziehungsweise im Zaum halten könne. In Luzern ist zunächst die Erwartungshaltung der Touristen, also eigentlich die Fremdbilder, handlungsleitend, die dazu führt, Luzern als Ausgangspunkt in die Schweizer Bergwelt und zum Vierwaldstätter See zu akzentuieren. Die touristische Identität ist hier vollkommen prägend, Gegenstimmen werden zwar artikuliert, setzen sich jedoch praktisch nicht durch. Dagegen ist die Identität von Bern wohl längerfristig unklar und kontrovers, was auch explizit von Stadt- und Reiseführern thematisiert wird. Man spricht von verschiedenen Gesichtern der Stadt als Bauernstadt, Hauptstadt und Weltstadt. Sehr schön kann Fasol diese Heterogenität auch an verschiedenen, rasch wechselnden Gestaltungen des Titelbilds des Berner Fremdenblatts zeigen. Der Bezug zur Geschichte war in Bern am ausgeprägtesten, hier wurde insbesondere die Altstadt im Ganzen als historisches Zeugnis präsentiert, während in Luzern die Altstadt zunehmend weniger wichtig erschien, im Vergleich zum Touristenquartier; nur die Kapellbrücke wird - hier auch verstärkt aufgrund des kollektiven Protests englischer Gäste gegen einen befürchteten Abbruch - als historisches Wahrzeichen verstanden.

Deutlich unterschiedlich ist die Haltung zur Moderne in der Konstruktion von Stadtidentitäten. Während sich Winterthur klar zum industriegesellschaftlichen Fortschritt bekennt, der allerdings durch gartenstadtähnliche Stadtentwicklung in seinen Auswirkungen gezähmt werden soll, ist in Bern das affirmative Bekenntnis zur ,Langsamkeit‘ Ausdruck einer prinzipiellen Fortschrittsskepsis, die sich auch in planerischer Regulierung, etwa des Kirchenfeldareals, industriekritisch artikulierte. Natur war in allen drei Städten wichtig, doch ihr Inhalt zeigt sich jeweils unterschiedlich. Winterthur präsentiert sich als moderne Industriestadt eingebettet in eine liebliche Landschaft. Luzern zeigt sich als Ausgangspunkt in die Alpenwelt, die in den jüngeren Stadtveduten den eigentlichen Bezugspunkt bildet. In Bern wird die Stadt selbst deutlich stärker akzentuiert, aber das Alpenpanorama ist zugleich konstitutiver Bildbestandteil.

Die Argumentation von Fasol zur Konstruktion der Stadtidentitäten kann insgesamt überzeugen, die Quellen werden sehr gut kontextualisiert. Allerdings hätte man im Hinblick auf eine stärkere Integration der Schweizer Stadtgeschichte in die europäische historische Stadtforschung gerne noch Bezüge zu Referenzwerken, wie Wolfgang Behringer/Bernd Roeck: Das Bild der Stadt, gesehen. Auch eine Reflexion zur Erklärungskraft des ursprünglich stadtsoziologischen, aber auch in der Stadtgeschichte rezipierten Ansatzes ,Eigenlogik der Städte“ (Martina Löw/Helmuth 
Berking) hätte sich insbesondere beim Fallbeispiel Bern mit seinem Akzent auf habituellen Mustern wie der ,Langsamkeit‘ geradezu aufgedrängt. Und für nichtschweizerische Leser wären kurze Erläuterungen zu Spezifika wie etwa der Berner ,Burgergemeinde" hilfreich gewesen. Dennoch leistet die Studie von Laura Fasol einen überzeugenden, gut geschriebenen und hervorragend bebilderten Beitrag zur vergleichenden Forschung zu Stadtbildern und Stadtidentitäten.

Funding Open Access funding enabled and organized by Projekt DEAL.

Open Access Dieser Artikel wird unter der Creative Commons Namensnennung 4.0 International Lizenz veröffentlicht, welche die Nutzung, Vervielfältigung, Bearbeitung, Verbreitung und Wiedergabe in jeglichem Medium und Format erlaubt, sofern Sie den/die ursprünglichen Autor(en) und die Quelle ordnungsgemäß nennen, einen Link zur Creative Commons Lizenz beifügen und angeben, ob Änderungen vorgenommen wurden.

Die in diesem Artikel enthaltenen Bilder und sonstiges Drittmaterial unterliegen ebenfalls der genannten Creative Commons Lizenz, sofern sich aus der Abbildungslegende nichts anderes ergibt. Sofern das betreffende Material nicht unter der genannten Creative Commons Lizenz steht und die betreffende Handlung nicht nach gesetzlichen Vorschriften erlaubt ist, ist für die oben aufgeführten Weiterverwendungen des Materials die Einwilligung des jeweiligen Rechteinhabers einzuholen.

Weitere Details zur Lizenz entnehmen Sie bitte der Lizenzinformation auf http://creativecommons.org/ licenses/by/4.0/deed.de.

\section{Borio, Claudio u.a. (Hrsg.): Promoting Global Monetary and Financial Stability. The Bank for International Settlements after Bretton Woods, 1973-2020, 302 S., Cambridge UP, Cambridge 2020.}

\section{Matthias Kemmerer}

Angenommen: 29. April 2021 / Online publiziert: 22. Mai 2021

(C) Der/die Autor(en) 2021

Die Bank für Internationalen Zahlungsausgleich (BIZ) diente bei ihrer Gründung 1930 dem Zweck, die deutschen Reparationszahlungen nach dem Ersten Weltkrieg neu zu regeln. Mittlerweile ist die in Basel ansässige ,Bank der Zentralbanken“ eine internationale Finanzorganisation, die die globale Währungs- und Finanzstabilität

M. Kemmerer $(\bowtie)$

Goethe-Universität Frankfurt am Main, Frankfurt a. M., Deutschland

E-Mail: matthias.kemmerer@stud.uni-frankfurt.de 